\title{
Optical, wave measurements, and modeling of helicon plasmas for a wide range of magnetic fields
}

\author{
Shane M. Tysk, C. Mark Denning, John E. Scharer, and Kamran Akhtar \\ Department of Electrical and Computer Engineering, University of Wisconsin-Madison, \\ Madison, Wisconsin 53706
}

(Received 2 October 2003; accepted 24 November 2003)

\begin{abstract}
Helicon waves are excited in a plasma wave facility by a half-turn double-helix antenna operating at $13.56 \mathrm{MHz}$ for static magnetic fields ranging from 200 to $1000 \mathrm{G}$. A non-perturbing optical probe located outside the Pyrex ${ }^{\mathrm{TM}}$ plasma chamber is used to observe $443 \mathrm{~nm}$ Ar II emission that is spatially and temporally correlated with the helicon wave. The Ar II emission is measured along with wave magnetic and Langmuir probe density measurements at various axial and radial positions. $105 \mathrm{GHz}$ interferometry is used to verify the bulk temperature corrected Langmuir probe measurements. The measured peak Ar II emission phase velocity is compared to the measured wave magnetic field phase velocity and code predicted wave phase velocity for the transition and blue mode regimes. Very different properties of the optical emission peak phase and wave characteristics for the transition and helicon modes of operation are observed. Comparison of the experimental results with the ANTENAII code [Y. Mouzouris and J. E. Scharer, IEEE Trans. Plasma Sci. 24, 152 (1996)] is carried out for the wave field measurements for the two regimes of operation. (C) 2004 American Institute of Physics. [DOI: 10.1063/1.1642656]
\end{abstract}

\section{INTRODUCTION}

Helicon sources are a class of highly efficient inductively coupled plasma sources that cover a wide range of plasma parameters. The standard configuration for this type of source is a cylindrical geometry with an axial magnetic field and one of many types of rf antennae operating at a frequency $\omega$ between the ion cyclotron frequency $\left(\omega_{c i}\right)$ and the electron cyclotron frequency $\left(\omega_{c e}\right)$. Unlike other inductive sources, the helicon source ionizes the neutral target gas through the propagation and absorption of helicon and Trivelpiece-Gould plasma waves excited by the antenna. These sources are capable of producing moderate- to highdensity, highly ionized $(4 \%-40 \%)$ plasmas with less than 2 $\mathrm{kW}$ of rf power. Typically, these sources operate with axial fields in the 20-2000 $\mathrm{G}$ range and densities ranging from $10^{11}$ to $10^{14} \mathrm{~cm}^{-3}$, thus admitting a wide variety of potential physical processes. In recent years the physical processes resulting in highly efficient helicon source operation ${ }^{1,2}$ have been extensively studied over a wide variety of operating regimes. Collisional processes, ${ }^{1,2}$ Landau damping, ${ }^{2-4}$ helicon wave penetration, ${ }^{5}$ antenna localized acceleration, ${ }^{6,7}$ mode conversion near the lower hybrid frequency, ${ }^{8}$ nonlinear trapping of fast electrons, ${ }^{9,10}$ and ion heating ${ }^{11}$ have been examined for different operating regimes. The effects of a substantial axial variation in the source static magnetic field and electron temperature anisotropy have also been measured and modeled. ${ }^{12}$

These plasma sources are of considerable interest in a wide range of applications including sub-micron semiconductor wafer etching ${ }^{13,14}$ and integrated fiber optics circuit fabrication (e.g., splitters and combiners in substrates). ${ }^{15}$ They have also been used for plasma confinement studies, space-plasma simulation experiments, ${ }^{16}$ and are of substan- tial interest as part of the VASIMR thruster engine for Mars space vehicles. ${ }^{17-19}$

Recent studies of helicon sources have focused on the physics behind their highly efficient ionization and strong wave damping, which is not fully explained by either collisional or Landau damping processes. The significance of the role of a population of fast electrons constituting a nonMaxwellian component of the electron distribution function in the helicon ionization process is a central question. These processes influence silicon etching since fast electrons and ion heating degrade etching performance. However, fast electrons can give rise to enhanced rf skin effect penetration that can enhance plasma uniformity and improve plasma thruster applications. Evidence of non-Maxwellian electrons were observed by Molvik et al. ${ }^{20}$ using a gridded electron energy analyzer in a Nagoya Type-III antenna helicon plasma source $\left(P=1.3-3.3 \mathrm{~kW}, B_{0}=90-30 \mathrm{G}\right.$, diameter $=15 \mathrm{~cm}$ ). Alternatively, Maxwellian distributions were observed by Blackwell and $\mathrm{Chen}^{21}$ in a Nagoya Type-III helicon plasma source $\left(P=1 \mathrm{~kW}, \quad B_{0}=360 \mathrm{G}\right.$, diameter $=10 \mathrm{~cm}$ ). It should be noted that these experiments were carried out under substantially different plasma conditions, illustrating that helicon source ionization mechanisms can be sensitively dependent on the plasma density, magnetic field, and wave power.

In recent research on the WOMBAT helicon facility ${ }^{22}$ with moderate densities $\left(n_{\max }=1.2 \times 10^{12} \mathrm{~cm}^{-3}\right)$ and low magnetic field $\left(B_{0}=100 \mathrm{G}\right)$, observations of correlated peak phase, excited state $443 \mathrm{~nm}$ Ar II optical emission and wave magnetic field phase velocities were presented. The wave field measurements were modeled by a one-dimensional (1-D) acceleration code that illustrated substantial increases of the electron energy distribution function above Maxwell- 


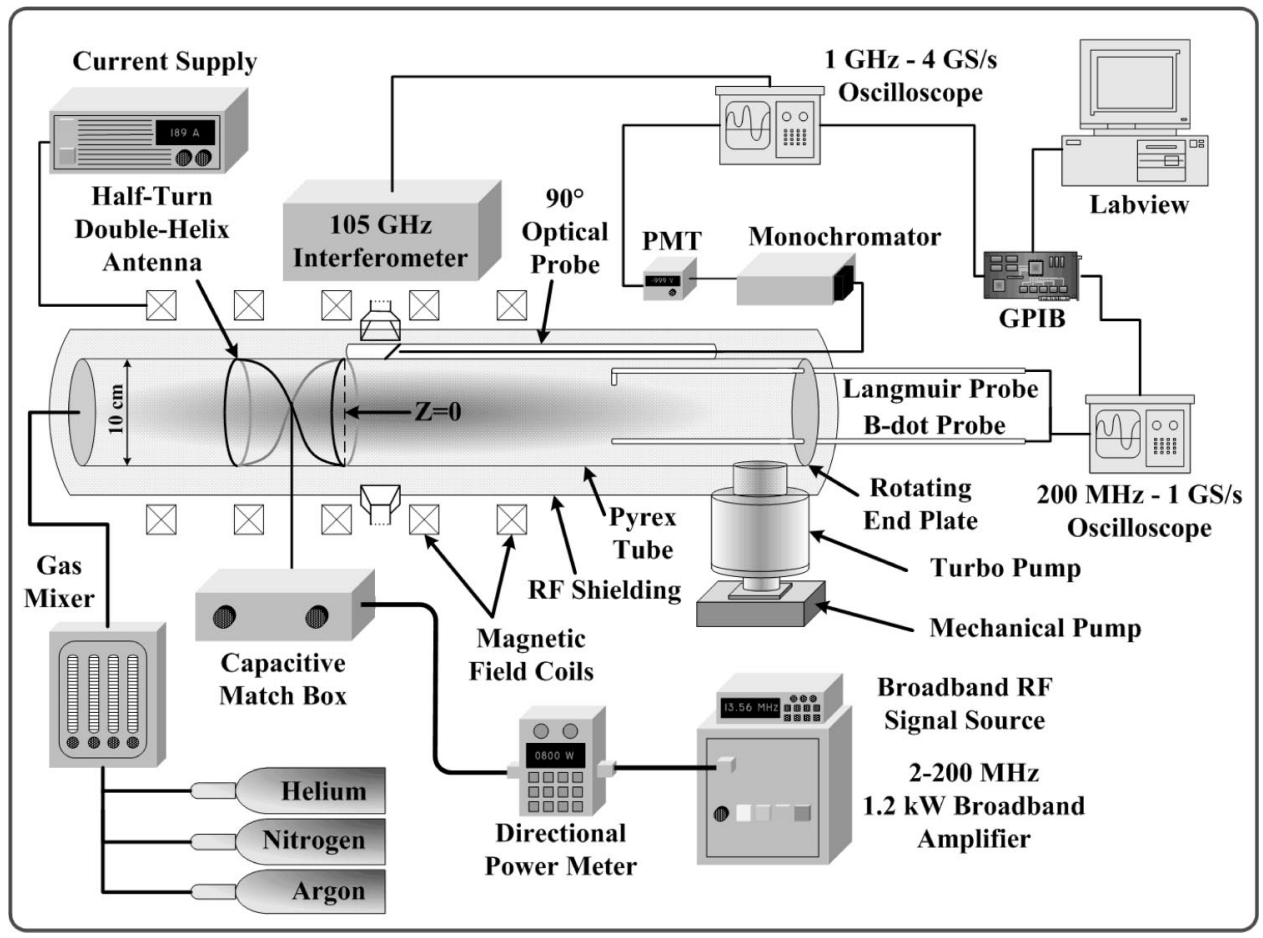

FIG. 1. Schematic diagram of the helicon plasma facility.

ian levels in the 15-35 eV energy range, which enhance the ionization rate compared to a Maxwellian distribution. These energies were found to be comparable to the energies of resonant electrons, traveling at the wave phase velocity.

In current experiments on our helicon plasma wave facility, we have created higher density plasmas $\left(n_{\max }\right.$ $=0.5-3 \times 10^{13} \mathrm{~cm}^{-3}$ ) for a wide range of magnetic fields $\left(B_{0}=200-1000 \mathrm{G}\right)$. A half-turn double-helix antenna is utilized, which primarily excites the $m=+1$ azimuthal mode. The source exhibits three distinct stable operating regimes. ${ }^{23}$ The first, a low-density $\left(n_{e} \simeq 10^{11} \mathrm{~cm}^{-3}\right)$ inductive regime $\left(P<650 \mathrm{~W}, B_{0}<200 \mathrm{G}\right)$, is characterized by an evanescent wave structure peaked under the antenna. The second, a moderate-density $\left(n_{e} \simeq 10^{12} \mathrm{~cm}^{-3}\right)$ transition regime $(P$ $\left.=650-900 \mathrm{~W}, B_{0}=200-400 \mathrm{G}\right)$, is characterized by a dramatic increase in density with small changes in power and magnetic field and a traveling wave structure. The third, a high-density $\left(n_{e} \simeq 10^{13} \mathrm{~cm}^{-3}\right)$ helicon regime $(P>700 \mathrm{~W}$, $B_{0}>400 \mathrm{G}$ ), is characterized by a $4 \mathrm{~cm}$ diam intense "blue core" plasma with a complex wave structure. In the transition and helicon regimes, substantial downstream density and emission peaks have been observed. The objective of this paper is to present measurements of the plasma properties and clarify the physical processes that influence helicon source operation over a wide range of magnetic field conditions.

\section{EXPERIMENTAL FACILITY}

The University of Wisconsin helicon plasma facility shown in Fig. 1 consists of a 10-cm-i.d. Pyrex ${ }^{\mathrm{TM}}$ plasma chamber surrounded by magnetic coils used to produce the dc magnetic field. Argon gas is fed into the chamber from the upstream side of the $1.75 \mathrm{~m}$ chamber so that the gas flows through the antenna source region. Previous research ${ }^{22}$ on the WOMBAT experiment fed the argon gas to the system from the downstream large diffusion chamber, reducing the neutral gas feed to the source for ionization compared to the present case. The downstream end of our chamber has dry mechanical and turbo-molecular pumps that can achieve a base pressure of $10^{-6}$ Torr and maintain the argon gas pressure at 3 mTorr. The plasma is created with $13.56 \mathrm{MHz}$ rf that is pulsed for $6 \mathrm{~ms}$ with a $10 \%$ duty cycle. The rf is matched to the 20-cm-long half-turn double-helix antenna with a very efficient, variable capacitor matching network capable of reducing the reflected power at $13.56 \mathrm{MHz}$ to less than $1 \%$ over the entire operating range of interest. The primary diagnostic tools used to examine the plasma are a Langmuir probe, a magnetic field probe, a $105 \mathrm{GHz}$ interferometer, and an optical probe. The diagnostic probes are shown in Fig. 1 and in more detail in Figs. 2 and 3.

The rf-compensated Langmuir probe has a three stage radiofrequency choke at the fundamental (f), second (2f), and third harmonics (3f), which reduced harmonics by 42.3, 17.4, and $17.6 \mathrm{~dB}$, respectively, to ensure a more accurate measurement of plasma density and bulk temperature. The probe filtering does not allow time varying, non-Maxwellian properties of the electron energy distribution to be readily observed. The plasma density was measured with the cylindrical Langmuir probe using ion saturation current measurements where the Bohm velocity is determined using the axial bulk electron temperature measurements. ${ }^{24}$ Density measurements are then cross-checked with the interferometer incorporating radial profile effects.

The magnetic field probe is a 2-mm-diam, six-turn helical antenna enclosed in a 35-cm-long, $\frac{1}{4}$-in.-o.d. Pyrex tube. The hybrid combiner [Fig. 2(a)] used to separate the in-phase and out-of-phase signals was measured to have a common mode rejection ratio of $34.7 \mathrm{~dB}$. The frequency response of 


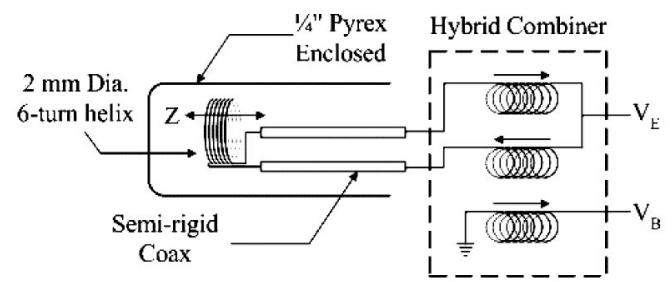

(a)

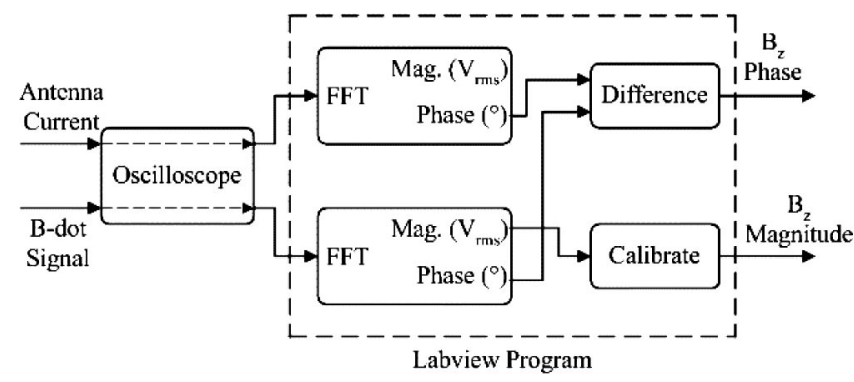

(b)

FIG. 2. A schematic diagram of (a) the magnetic field probe (arrows indicate sense of wiring around ferrite toroid) and (b) magnetic field probe data acquisition and analysis.

the hybrid combiner was measured to be relatively flat $( \pm 0.5 \mathrm{~dB})$ for frequencies up to $40 \mathrm{MHz}$. The magnetic field probe is calibrated using a Helmholtz coil, a current monitor, and a spectrum analyzer.

The optical probe consists of a $45^{\circ}$ optical mirror that directs the plasma emission into a fiber optic cable that feeds a $0.5 \mathrm{~m}$ monochromator (Figs. 1 and 3 ). The monochromator

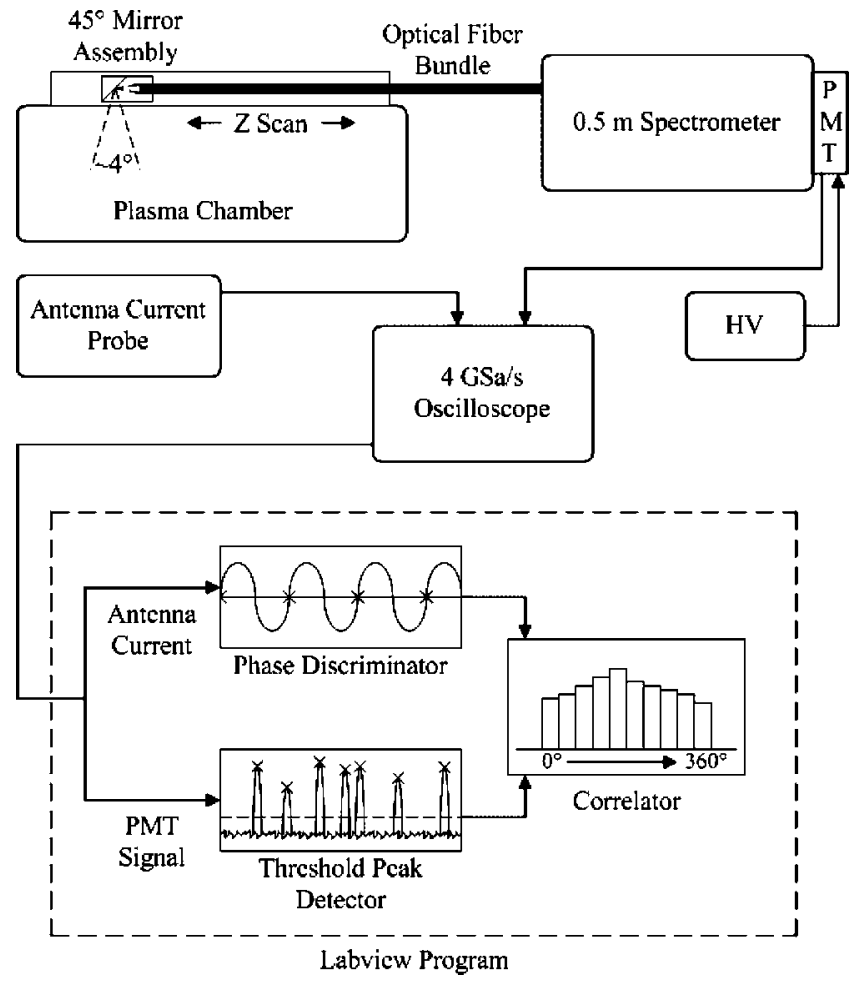

FIG. 3. Optical probe data collection setup.

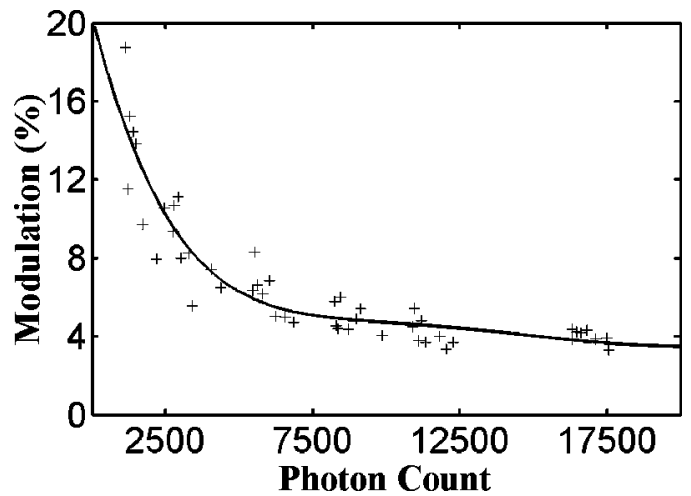

FIG. 4. Threshold for statistically significant modulation as a function of photons acquired per acquisition.

is set up to observe $443 \mathrm{~nm}$ emission produced by the plasma. This emission line corresponds to an excited state of singly ionized argon located $35 \mathrm{eV}$ above the neutral ground state with a 6.92 ns lifetime. ${ }^{22}$ The output photo-multiplier tube (PMT) on the monochromator is used to count photons as they are emitted from the plasma. Through the use of computer software and a $4 \mathrm{GSa} / \mathrm{s}$ oscilloscope, the photon emission during a $5 \mu$ s window was correlated with the phase of the antenna current feed that creates the plasma and counted in $7.3 \mathrm{~ns}$ period bins. The modulation index $(\mathrm{m})$ of the measured signal is given in Eq. (1) where $b_{i}$ is the photon count in the $i$ th bin, $b_{\max }$ is the maximum count, $b_{\min }$ is the minimum count, and $N$ is the total number of bins,

$$
m=\frac{b_{\max }-b_{\min }}{\frac{2}{N} \Sigma_{i} b_{i}} .
$$

Computer generated signals that replicate the photon emission and sampling rate of the oscilloscope were used to verify the proper operation of the program and determine a base level for statistically significant modulation (Fig. 4). Careful checks utilizing a random signal generator and phase shifting the optical signal when the plasma source was activated were carried out to ensure that there is minimal background rf coupling to the diagnostic and that the $443 \mathrm{~nm}$ background and modulation at the rf frequency arises from the plasma. Photon binning was also conducted at the end of the rf pulse and the modulation was observed to reduce to the random background level as the plasma relaxed over a $50 \mu \mathrm{s}$ period.

The $105 \mathrm{GHz}$ interferometer is a non-perturbing diagnostic used primarily to determine density. The interferometer is a quadrature phase, millimeter wave interferometer in a Mach-Zehnder configuration. The interferometer utilizes an $I-Q$ mixer to determine the amplitude and phase change of the $105 \mathrm{GHz}$ wave in the plasma arm of the interferometer.

\section{EXPERIMENTAL RESULTS}

The on-axis Langmuir probe peak density was compared to the line integrated density obtained from a $105 \mathrm{GHz} \mathrm{mm}$ wave interferometer and is shown in Fig. 5. Interferometer 


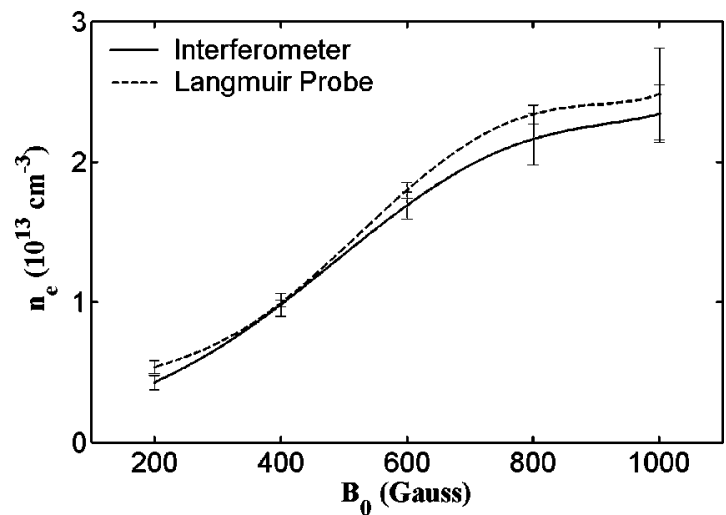

FIG. 5. Langmuir probe and interferometer peak density comparison at $r$ $=0 \mathrm{~cm}$ and $z=8 \mathrm{~cm}$.

and Langmuir probe comparison data were taken at $z=8 \mathrm{~cm}$ from the end of the antenna, and the magnetic field $\left(B_{0}\right)$ was scanned from 200 to $1000 \mathrm{G}$. Comparison of the measurements was made at the temporal density peak occurring at $t \approx 0.5 \mathrm{~ms}$. Both amplitude and phase data from the interferometer are used since attenuation is non-negligible due to plasma density and collisionality. ${ }^{25}$ Central density was deduced from interferometer results using radial density profiles measured with the Langmuir probe. It should be noted that both the Langmuir probe results and the collisional model for the ordinary mode $\left(\mathrm{O}\right.$-mode, $\left.\mathbf{E} \| \mathbf{B}_{0}\right)$ interferometer data analysis assume a Maxwellian electron distribution, and therefore they do not account for nonMaxwellian electron distribution effects.

Figure 6 shows the plasma density radial profile as a function of magnetic field and wave power measured at $z$ $=15 \mathrm{~cm}$. Note that the density profile becomes highly peaked near the axis as the magnetic field is increased to $1000 \mathrm{G}$, showing that the helicon mode is characterized by a high, centrally peaked radial density profile that implies a wave absorption and ionization process that is concentrated in the plasma core at higher magnetic fields. Axial density profiles measured at the radial center of the plasma chamber are shown in Fig. 7 at $t=1.5 \mathrm{~ms}$.

Figure 8 details the on-axis plasma density evolution versus position and time as the plasma parameters are changed from the inductive to transition to helicon modes. All data are taken at the center of the plasma chamber $(r$ $=0$ ) where the density peaks, with the magnetic field probe removed. The density increases from the $10^{11} \mathrm{~cm}^{-3}$ range to $10^{12} \mathrm{~cm}^{-3}$ in the transition and finally to the $10^{13} \mathrm{~cm}^{-3}$ range for full helicon mode. The transition mode shows a density peak away from the antenna at $z \approx 16 \mathrm{~cm}$ and the helicon mode peaks near the same axial position but with less axial variation. In Figs. 7 and 8(b) $-8(d)$ a spatial expansion of the high density region is observed as the magnetic field is increased at constant power. In addition, the high density region can be seen to persist for an extended period of time at the higher magnetic fields as seen in Figs. 8(c) and $8(\mathrm{~d})$ relative to Fig. $8(\mathrm{~b})$. The dc axial magnetic field $\left(B_{0}\right)$ is uniform within $5 \%$ from $z=-20 \mathrm{~cm}$ to $z=28 \mathrm{~cm}$ and drops to $66 \%$ of the uniform value at $z=-32 \mathrm{~cm}$ and $z=40 \mathrm{~cm}$
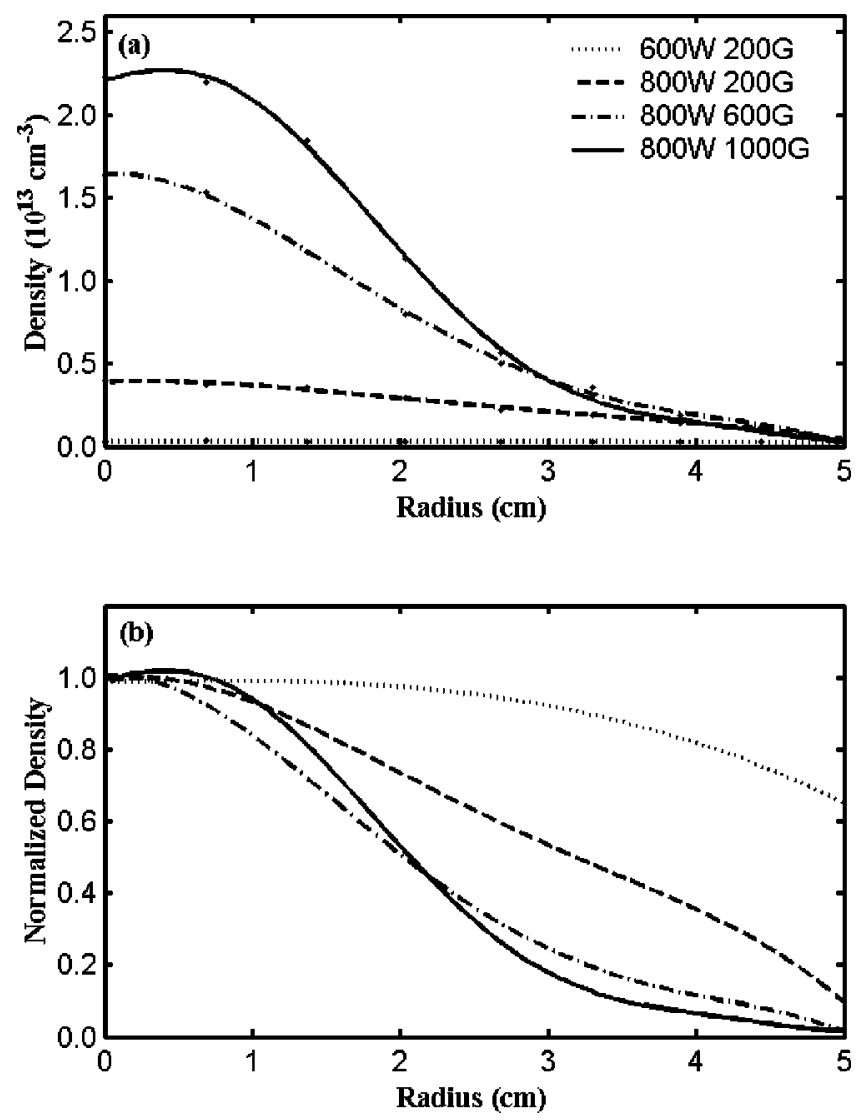

FIG. 6. Radial profiles measured at $z=15 \mathrm{~cm}$ for specified powers and magnetic field strengths given (a) in units of $10^{13} \mathrm{~cm}^{-3}$ and (b) normalized units.

resulting in significant density reduction beyond those points.

Figure 9 details Langmuir probe bulk axial electron temperature measurements ${ }^{26}$ for two representative cases at $r$ $=0$. The transition mode case of $800 \mathrm{~W}, 200 \mathrm{G}$ shows a relatively flat axial temperature profile of $4.3 \mathrm{eV}$ that increases near the antenna. The helicon mode case of $800 \mathrm{~W}$, $1000 \mathrm{G}$ shows a similarly shaped axial profile with a reduced

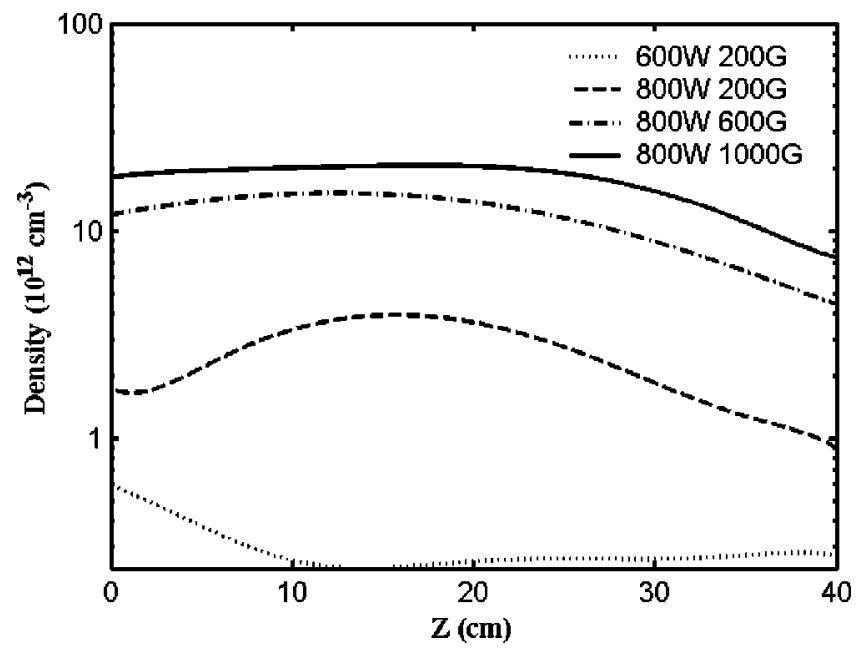

FIG. 7. Axial profiles measured at $r=0 \mathrm{~cm}$. 


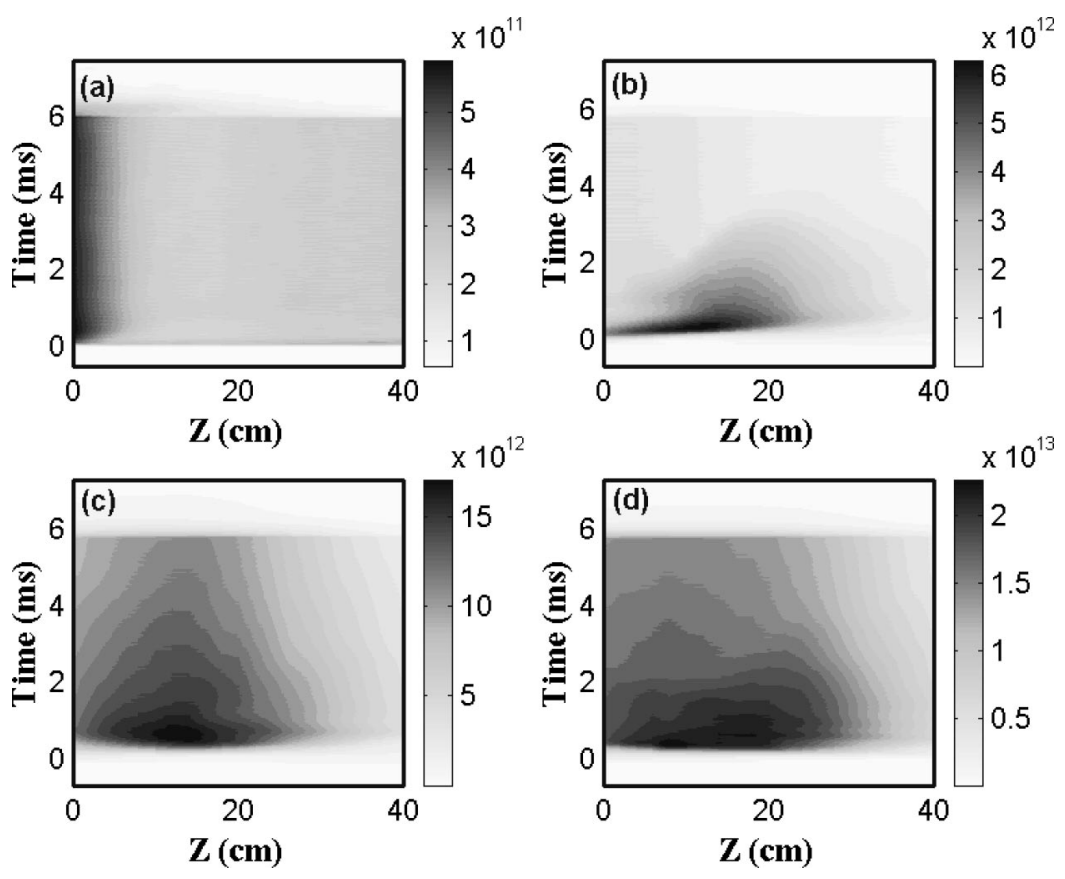

FIG. 8. Spatio-temporal plots of plasma density $\left(\mathrm{cm}^{-3}\right)$ corrected for local electron temperature for the (a) inductive mode- $600 \mathrm{~W}, 200 \mathrm{G}$, (b) transition mode$800 \mathrm{~W}, 200 \mathrm{G}$, (c) helicon mode- $800 \mathrm{~W}, 600 \mathrm{G}$, and (d) helicon mode- $800 \mathrm{~W}, 1000 \mathrm{G} . \mathrm{Z}(\mathrm{cm})$ is measured from the end of the antenna.

bulk temperature of $3.5 \mathrm{eV}$ over the flat interval region. Bulk temperature profiles showed little radial variation, consistent with other helicon measurements. ${ }^{27}$

The magnetic field probe was inserted into the plasma at a radius of $3.25 \mathrm{~cm}$. Careful simultaneous measurements of the axial plasma density profile and wave $B_{z}$ field were made to minimize the effects of probe perturbations on the plasma and wave characteristics. The presence of the magnetic field probe does not substantially alter the characteristic of the density profile. Using the antenna current as a reference [Fig. 2(b)], the amplitude and phase of the plasma axial wave magnetic field at the fundamental frequency was obtained (Fig. 10). The data were taken at $1.5 \mathrm{~ms}$ into the pulse where Langmuir probe results showed that the plasma is in quasisteady state.

Several properties are observed from the magnetic field probe data presented in Fig. 10. First, the existence of the evanescent inductive mode at $600 \mathrm{~W}$ and $200 \mathrm{G}$ is very clearly evident by the flat phase and decaying amplitude in Fig. 10(a). When the power is increased to $800 \mathrm{~W}$ and the magnetic field is held constant, the plasma changes to the

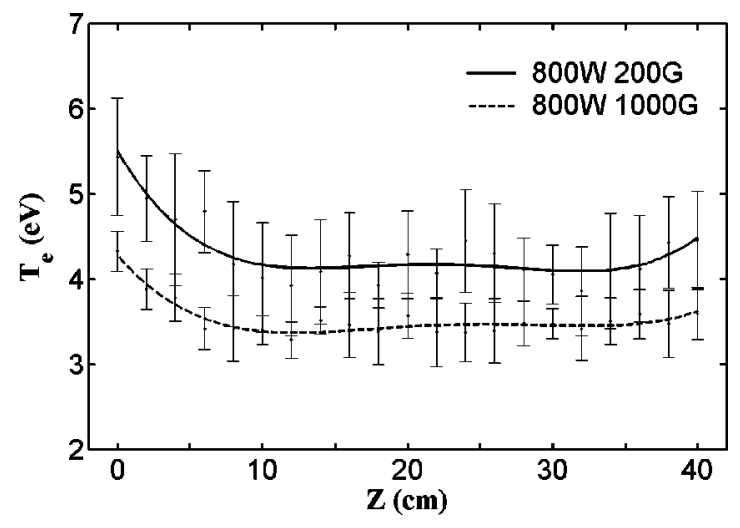

FIG. 9. Langmuir probe bulk electron temperature measurements. transition mode. As shown in Fig. 10(b), the field has a traveling wave characteristic as shown by the smoothly increasing phase and relatively monotonic variation in wave amplitude versus position. Finally, Figs. 10(c) and 10(d) show the
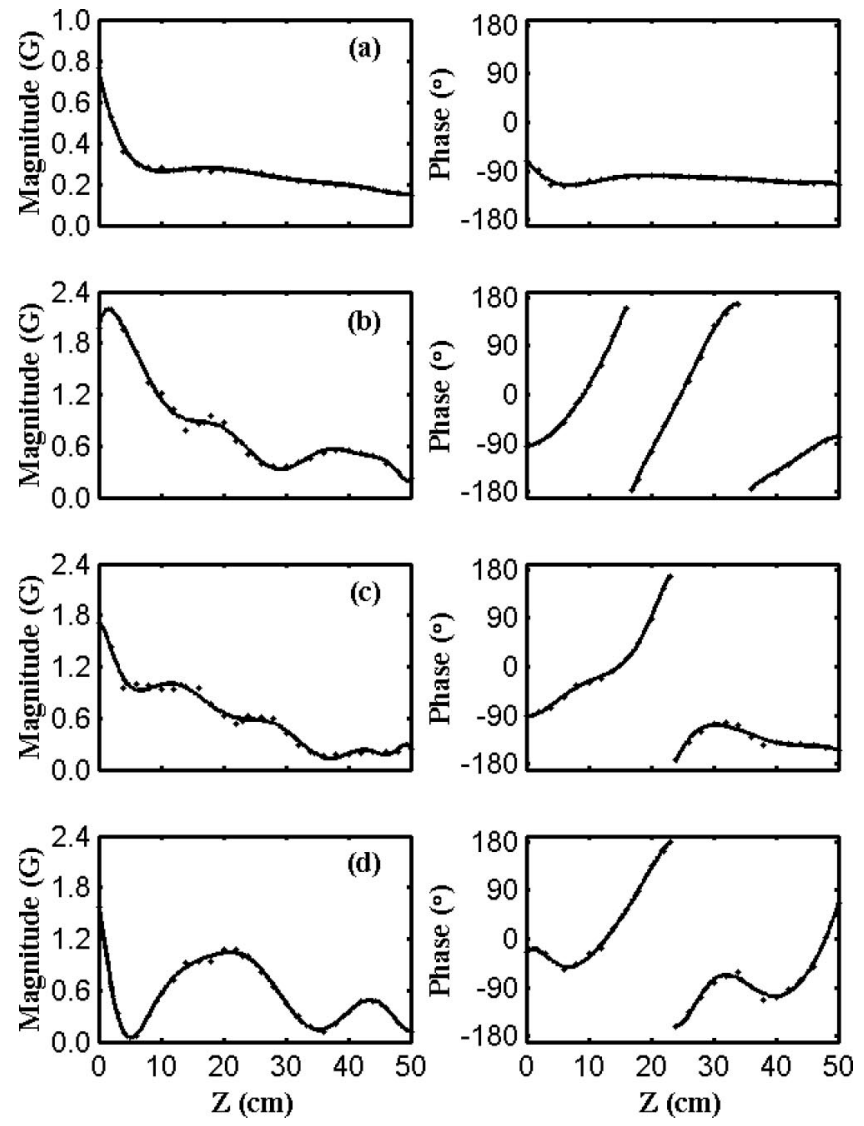

FIG. 10. $B_{z}$ magnitude and phase for (a) inductive mode- $600 \mathrm{~W}, 200 \mathrm{G}$, (b) transition mode-800 W, $200 \mathrm{G}$, (c) helicon mode-800 W, $600 \mathrm{G}$, and (d) helicon mode-800 W, $1000 \mathrm{G}$ at $t=1.5 \mathrm{~ms}$ and $r=3.25 \mathrm{~cm}$. 

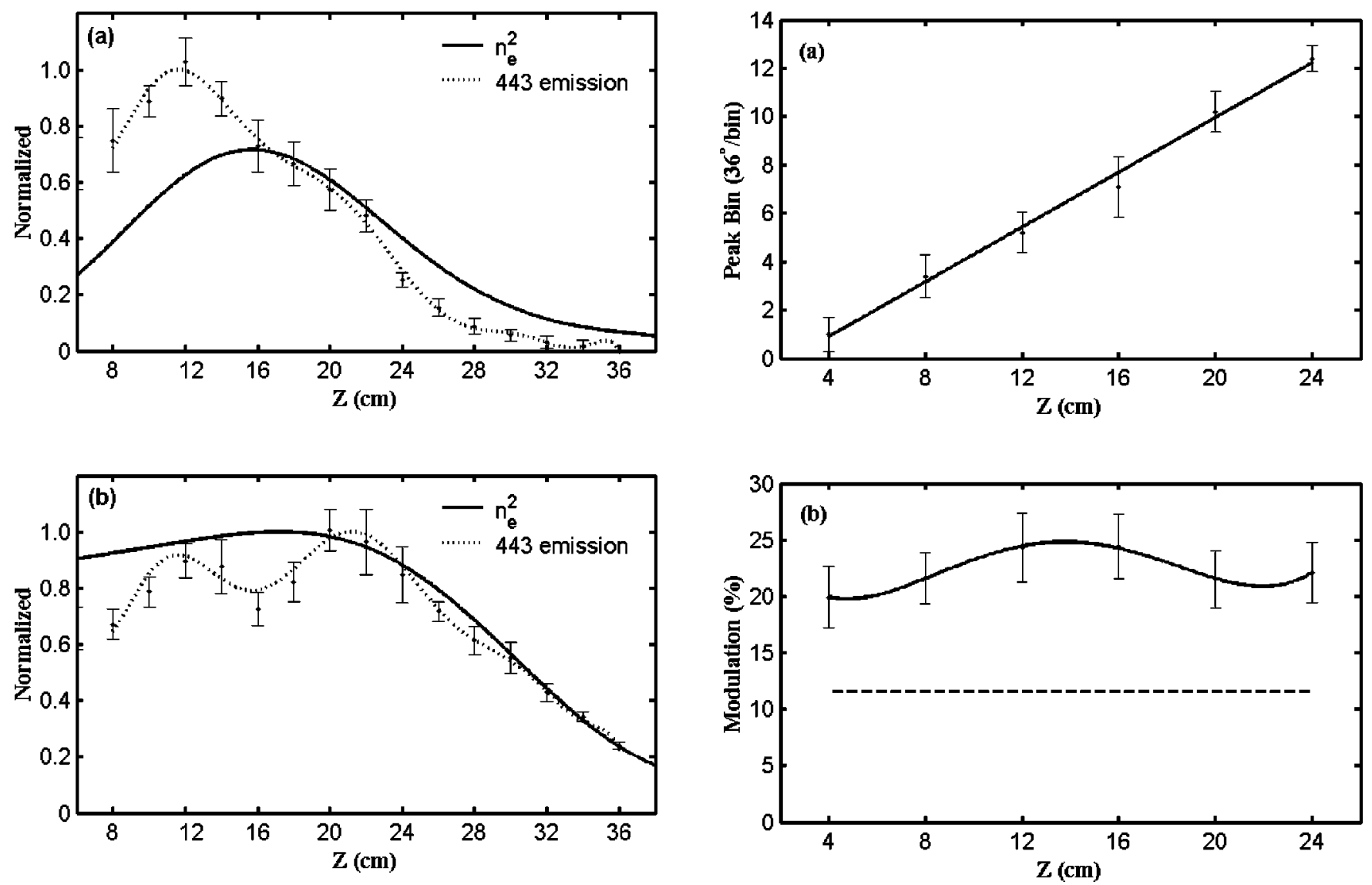

FIG. 11. Normalized axial emission and density comparison for (a) $800 \mathrm{~W}$, $200 \mathrm{G}$ case and (b) $800 \mathrm{~W}, 1000 \mathrm{G}$ case.

case where the magnetic field is increased to 600 and 1000 $\mathrm{G}$, respectively, with the power held constant at $800 \mathrm{~W}$. Under these conditions the plasma is said to be in the helicon "blue" mode. It is observed that the wave amplitude has a much higher standing wave ratio with substantial minima, and the wave phase exhibits smooth oscillations in the axial direction.

The frequency calibrated wave magnetic field probe was also used to examine electromagnetic rf wave harmonics. Semi-rigid coax (Fig. 2) minimizes capacitive pickup from the plasma and substantially reduces harmonic pickup. ${ }^{28}$ For all cases measured at positions from $z=6 \mathrm{~cm}$ near the antenna out to $z=30 \mathrm{~cm}$ from the end of the antenna, the first and second harmonics were at least 11 and $20 \mathrm{~dB}$ below the fundamental, respectively. The smaller relative harmonic amplitudes measured by the magnetic field probe are comparable to that measured by the loop probe at the driving antenna, indicating that they are driven by the antenna, rather than produced within the plasma by non-linear processes.

\section{OPTICAL DIAGNOSTIC RESULTS}

Optical measurements are made of the $443 \mathrm{~nm}$ Ar II $\operatorname{line}^{22}$ utilizing the external optical probe and diagnostic system shown in Figs. 1 and 3. The resulting data are presented in Figs. 11-13. The first ionic state of Ar has a $15.76 \mathrm{eV}$ threshold above the neutral ground state. ${ }^{29}$ An excited metastable ionic state has a threshold $30 \mathrm{eV}$ above the ground

FIG. 12. Transition mode ( $800 \mathrm{~W}, 200 \mathrm{G})$ (a) peak bin data and (b) modulation data with a $5 \mu$ s window $1.5 \mathrm{~ms}$ into the pulse. The dashed line in (b) represents the base level for statistically significant modulation.

state. The upper state for $443 \mathrm{~nm}$ emission is $35 \mathrm{eV}$ above the neutral ground state and is relatively isolated from other argon lines. Wave-electron interactions can excite the upper state from the neutral ground state, the first ionized state, or a metastable state directly resulting in $443 \mathrm{~nm}$ emission. Therefore, a $443 \mathrm{~nm}$ emission peak well-correlated with the rf drive phase is indicative of the presence of electrons in the range of $5-35 \mathrm{eV}$ that are accelerated by the wave.

The optical diagnostic measures $443 \mathrm{~nm}$ emission and phase correlates this emission with the rf wave on the antenna feed (See Sec. II and Fig. 3). The $443 \mathrm{~nm}$ excited state has a short lifetime $(6.92 \mathrm{~ns})^{22}$ relative to the rf wave period of $73.7 \mathrm{~ns}$. With adequate sampling $(4 \mathrm{GSa} / \mathrm{s})$, the rf period is resolved into ten bins. Equivalent photon counts in all bins are indicative of the absence of rf correlated effects. Conversely, modulation of the signal is indicative of rf correlated effects. Measurements were made during a $5 \mu$ s window, 1.5 $\mathrm{ms}$ into the rf pulse where the plasma is in a quasi-steady state. The external optical probe was moved axially to track $443 \mathrm{~nm}$ emission $\mathrm{rf}$ correlation effects. Comparisons of peaks in modulation with the local phase of the wave magnetic field from an axially scanned magnetic field probe enable a correlation between the plasma wave fields and electronic excitation emission to be observed.

Each data point consists of the required number of acquisitions needed to reach a threshold photon count to get 

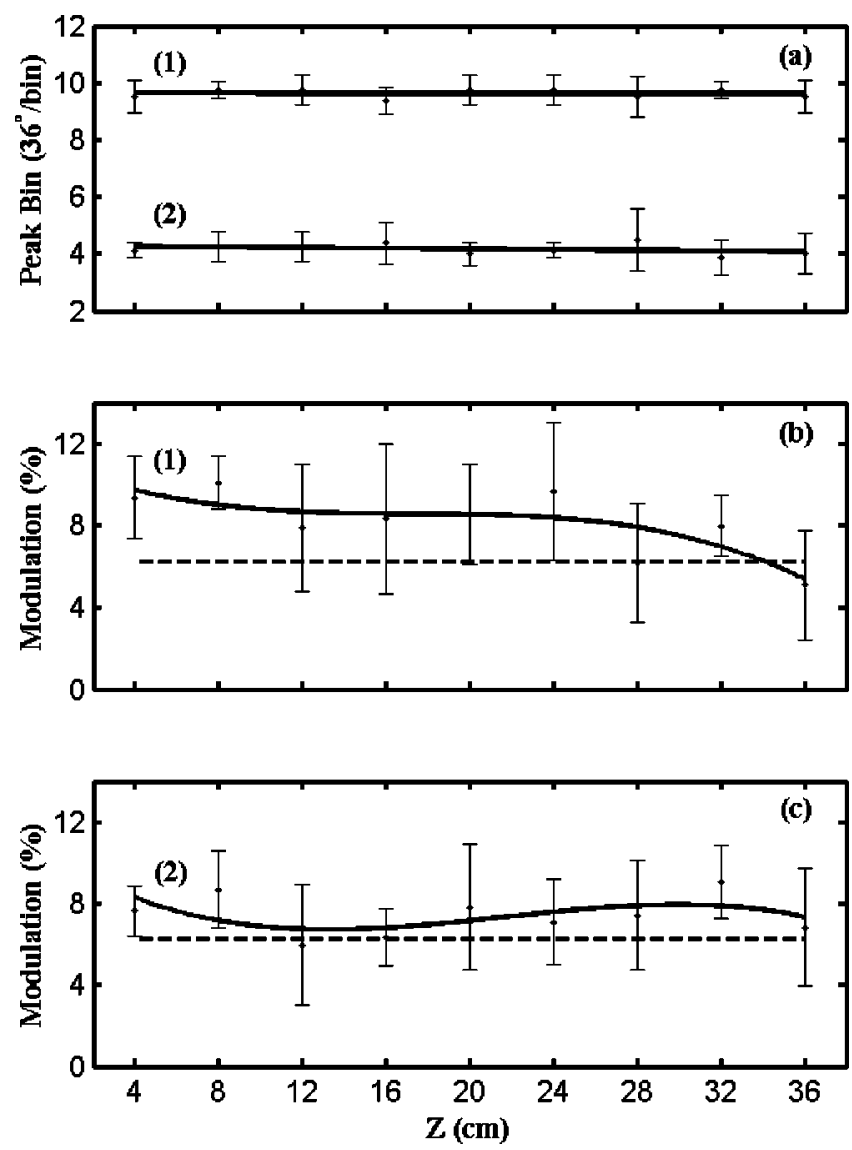

FIG. 13. Helicon mode ( $800 \mathrm{~W}, 1000 \mathrm{G})$ (a) peak bin data and (b) and (c) modulation data given separately for each peak as indicated by numbers (1 and 2) with a $5 \mu$ s window $1.5 \mathrm{~ms}$ into the pulse. The dashed lines in (b) and (c) represent the base level for statistically significant modulation.

statistically significant modulation (Fig. 4). A voltage divider circuit with decoupling capacitors on the PMT is utilized to increase the pulse output linearity. Also, a snubbing network is used to reduce the pulse fall time and decrease ringing effects below the observation threshold, which would register as extraneous photon counts in subsequent bins.

At a constant power of $800 \mathrm{~W}$, substantial $443 \mathrm{~nm}$ emission counts are observed during the $5 \mu$ s window at magnetic fields greater than $80 \mathrm{G}$. The transition from a diffuse pink plasma to an intense blue core (diameter $=4 \mathrm{~cm}$ ) plasma occurs near $400 \mathrm{G}$. The axial emission profile of the transition mode plasma $\left(B_{0}=200 \mathrm{G}\right)$ has a peak at $z$ $\approx 12 \mathrm{~cm}$ downstream from the antenna which is $4 \mathrm{~cm}$ upstream from the density peak at $z \approx 16 \mathrm{~cm}$ [Fig. 11(a)]. Emission from the intense blue core helicon mode plasma $\left(B_{0}\right.$ $=1000 \mathrm{G})$ has peaks at $z \approx 12 \mathrm{~cm}$ and $z \approx 20 \mathrm{~cm}$, neither of which correspond to the axial density peak at $z \approx 18 \mathrm{~cm}$ [Fig. $11(b)]$.

In the $200 \mathrm{G}$ lower axial magnetic field case, the $443 \mathrm{~nm}$ Ar II emission has a single strong binning peak during the rf period. The peak optical emission bin location moves axially as the optical probe is moved [Fig. 12(a)]. Modulation levels of 20\%-25\% are measured along the axis [Fig. 12(b)] in the transition regime indicating substantial wave-correlated emission with a maximum observed near the density peak at $z=15 \mathrm{~cm}$.
The phase velocity of the traveling emission peak is found to be $2.4 \times 10^{6} \mathrm{~m} / \mathrm{s}$ [Eq. (2)] corresponding to a resonant electron energy $\left(E=\frac{1}{2} m v_{\varphi}^{2}\right)$ of $16.4 \mathrm{eV}$,

$$
v_{\varphi 443}=\omega(\Delta \varphi / \Delta z)^{-1} \text {. }
$$

Over the axial range of $4-24 \mathrm{~cm}$ the simplified helicon dispersion relation can be written as $^{20}$

$$
v_{\varphi}=\frac{\omega}{k}=\left(\frac{\beta}{a}\right)\left(\frac{\omega_{c e} c^{2}}{\omega_{p e}^{2}}\right),
$$

where $a$ is the radius, $\beta$ is the radial mode number, $c$ is the speed of light, and $\omega_{c e}$ and $\omega_{p e}$ are cyclotron and plasma frequencies. This simplified dispersion relation predicts a phase velocity of $2.4 \times 10^{6} \mathrm{~m} / \mathrm{s}$ corresponding to a resonant electron energy of $16.4 \mathrm{eV}$ which agrees with the phase velocity of the traveling peak bin emission.

At axial magnetic field values greater than $400 \mathrm{G}$, an intense blue core of $4 \mathrm{~cm}$ diameter is observed. For both the $600 \mathrm{G}$ case and the $1000 \mathrm{G}$ case (Fig. 13) two strong phase stationary peaks $180^{\circ}$ out of phase are observed as the optical probe is moved axially. Modulation levels of $12 \%-23 \%$ are observed for the $600 \mathrm{G}$ case, significantly lower modulation levels of about $7 \%-10 \%$ are observed in the $1000 \mathrm{G}$ case. The decrease in modulation with increasing axial field suggests that modification of the electron distribution function from a Maxwellian is reduced as the magnetic field is increased. J.E.S. has previously observed a constant phase $443 \mathrm{~nm}$ emission peak ${ }^{30}$ at higher coupled rf powers $(P$ $\left.>2.9 \mathrm{~kW}, B_{0}=100 \mathrm{G}\right)$ in research done in collaboration with Degeling, Boswell, and Borg on the WOMBAT ${ }^{22}$ experiment.

\section{COMPUTATIONAL MODELING}

The linear 1-D ANTENAII code ${ }^{3}$ is used to model the experimental observations. The code calculates the threedimensional electromagnetic plasma wave fields for a radially varying density in a cylindrical magnetized plasma using variable step integration. Maxwellian velocity distributions are assumed and Landau damping is included. Collisional effects are included via the Krook model ${ }^{3}$ and the total electron, ion, and neutral collision rates are calculated for each regime. ANTENAII models the helical antenna and boundary conditions present in the system. The antenna current $(I)$ input parameter is chosen to match the experimental input power $(P)$ of $800 \mathrm{~W}$, with less than $1 \%$ reflection, based on the relation $P=\frac{1}{2} I^{2} R_{A}$, where $R_{A}$ is the antenna radiation resistance as calculated by ANTENAII. We have used axially uniform densities corresponding to the average plasma density over the measurement region together with measured radial density profiles and bulk electron temperature.

Code runs were performed to model the transition (800 $\mathrm{W}, 200 \mathrm{G})$ and helicon $(800 \mathrm{~W}, 600 \mathrm{G}$, and $1000 \mathrm{G})$ modes of operation. Solutions that include the dominant fast electromagnetic helicon mode and the relatively negligible slow electrostatic Trivelpiece-Gould are obtained. A comparison of the computational and experimental results for the $B_{z}$ component of the plasma wave for these cases is given in Figs. 14-16. 

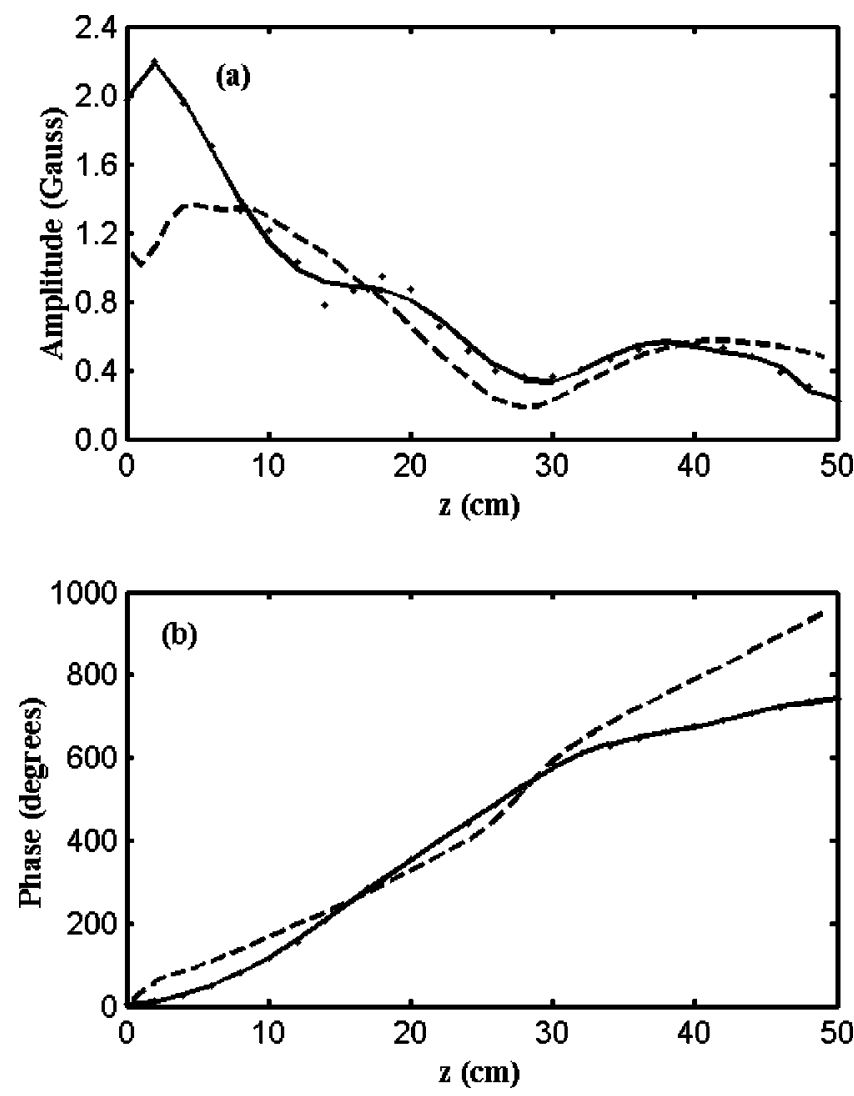

FIG. 14. Magnitude (a) and phase (b) of wave $B_{z}$ for transition mode (800 $\mathrm{W}, 200 \mathrm{G}$ ) of b-dot probe (solid) and ANTENAII (dashed) data.

For the transition mode, a good match (Fig. 14) is obtained only when the value for the effective collision frequency is increased by a factor of 5 above the thermal predicted value to $\nu=4 \times 10^{7} \mathrm{~Hz}$. The required enhancement of the effective collision frequency indicates that processes other than those provided by a Maxwellian distribution affect the wave magnetic fields.

A comparison of the transition mode $(800 \mathrm{~W}, 200 \mathrm{G})$ measured and ANTENAII modeled local phase velocity is given in Fig. 15. The comparison is obtained over the range of $z=4-24 \mathrm{~cm}$ where optical binning data were obtained. The measured $B_{z}$ field, calculated $B_{z}$ field, and peak optical

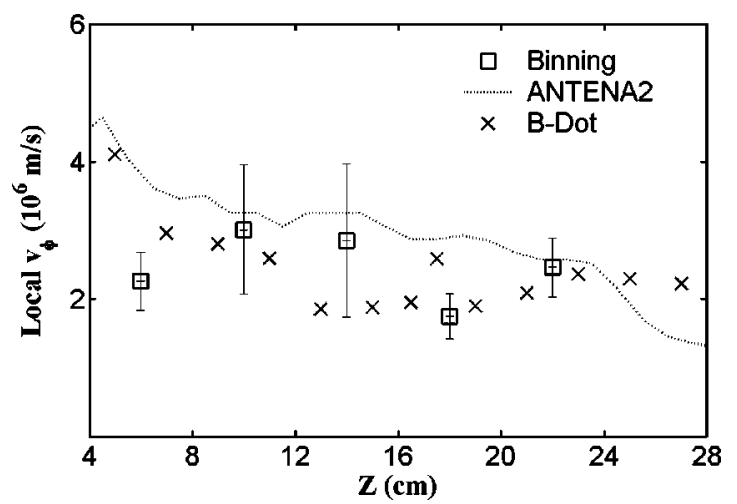

FIG. 15. Local phase velocity comparison for transition mode (800 W, 200 G).
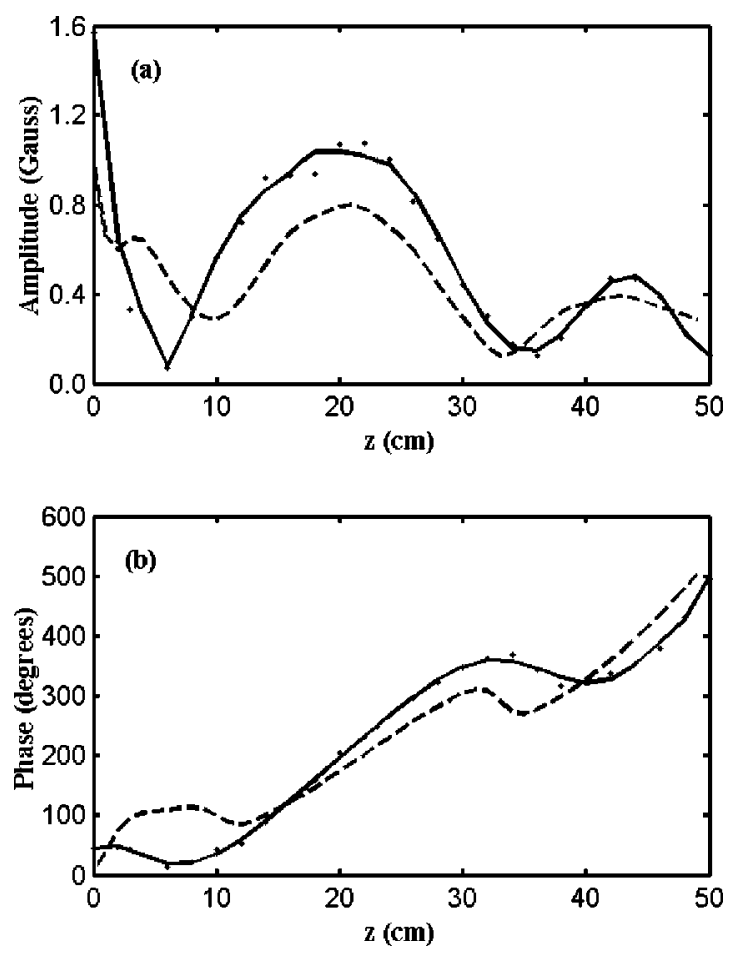

FIG. 16. Magnitude (a) and phase (b) of wave $B_{z}$ for blue mode (800 W, $1000 \mathrm{G}$ ) of B-dot probe (solid) and ANTENAII (dashed) data.

emission average phase velocities are $2.2 \times 10^{6}, 3.0 \times 10^{6}$, and $2.4 \times 10^{6} \mathrm{~m} / \mathrm{s}$, respectively, corresponding to an average resonant electron energy of $18 \mathrm{eV}$.

As shown in Fig. 16, the correlation with the measured amplitude and phase results are excellent for the high magnetic field "blue" mode. The density, temperature, and collision frequency corresponding to the measured plasma Maxwellian parameters provide good agreement with the experimental results for both the 600 and $1000 \mathrm{G}$ case. This close agreement indicates that for this mode the velocity distribution is close to a Maxwellian. Reduction of the density and magnetic field in code runs for the $200 \mathrm{G}$ case results in a reduced axial phase shift that agrees with the experimental observations for $z>35 \mathrm{~cm}$.

\section{ANALYSIS}

The experimental results shown in Figs. 7 and 8 indicate that the plasma source axial density characteristics change significantly for constant coupled rf power as $B_{0}$ is increased. The peak density is located well downstream $(z$ $=15-20 \mathrm{~cm}$ ) from the antenna, implying a non-local acceleration and ionization process. The density persistence is also longer for $B_{0}=600$ and $1000 \mathrm{G}$. The radial peaking of the density profile for $B_{0}=600$ and $1000 \mathrm{G}$ in Fig. 6 illustrates strong wave absorption and enhanced ionization peaked near the axis that correlates with the substantial axial attenuation of the wave magnetic field shown in Figs. 10(c) and $10(\mathrm{~d})$.

The substantial change in the $443 \mathrm{~nm}$ optical emission characteristics that occur for the more radially peaked, higher density and magnetic field "blue" mode regimes $(800 \mathrm{~W}$, 600 and $1000 \mathrm{G})$ is quite striking. The modulation in the 
optical emission will vary azimuthally as $\cos (\omega+m \theta)$, where the high density helicon mode fields are primarily due to the dominant $m=+1$ mode coupled to the plasma by the double-half-turn helix antenna. ${ }^{3}$ The corresponding axial electric field, $E_{z}$, for this mode can accelerate electrons traveling near the phase velocity of the wave. ${ }^{22}$ The $m=+1$ mode structure and strong radial density peak for the "blue" helicon mode accounts for the two comparable intensity peaks in emission separated by a $180^{\circ}$ time phase shift observed (Fig. 13) during a rf period. This is because the optical probe receives emission from the whole plasma diameter column. When the optical probe is tilted $10^{\circ}$ from the column central axis, a broader single strong peak with reduced modulation shifted in phase is observed in agreement with a dominant $m=+1$ azimuthal emission source. The broader radial density profile for the transition mode at $200 \mathrm{G}$ and $800 \mathrm{~W}$ shown in Fig. 6 indicates that the collected $443 \mathrm{~nm}$ emission for the radial source region located nearest the optical probe would be much stronger.

The ANTENAII modeling for the wave $B_{z}$ signals agrees quite well for the high density blue mode case $(800 \mathrm{~W}, 1000$ G) shown in Fig. 16 with the classical electron-ion collision rate, $\nu_{e i}$, obtained from the measured plasma density and temperature. This suggests the electron distribution is closer to a Maxwellian in this case. The ANTENAII modeling for the lower density transition mode $(800 \mathrm{~W}, 200 \mathrm{G})$, does not provide a good match utilizing the classical collision rate, $\nu_{e i}$. However, if the effective damping rate is enhanced by a factor of 5 above the classical rate, a good match with the wave $B_{z}$ field can be obtained as is shown in Fig. 14. This implies other processes that can include a non-Maxwellian electron distribution that enhances ionization can be present in this regime, as indicated in earlier research on the WOMBAT experiment. ${ }^{22}$ The traveling-wave peak of $443 \mathrm{~nm}$ optical emission and substantial modulation $(25 \%-20 \%)$ observed for the transition mode $(800 \mathrm{~W}, 200 \mathrm{G})$ indicates that wave acceleration of electrons near the phase velocity of the wave corresponding to resonant energies near $18 \mathrm{eV}$ play a substantial role in the argon ionization. The good correlation with the measured wave $B_{z}$ phase velocity and ANTENAII code modeling that requires an enhanced collision rate above the Maxwellian values further supports this view. Both results suggest that substantial modifications of the electron distribution function from a Maxwellian occur in the transition regime that can enhance ionization.

Another technique to determine whether the electron distribution is close to a Maxwellian is to compare the axial variation in $443 \mathrm{~nm}$ emission with the axial density profile. The Ar II ion optical emission will scale at the optical probe observation position if the electron distribution is Maxwellian as $I \sim\left\langle n_{e}(r, z)^{2} \sigma\left(T_{e}(r, z)\right)\right\rangle_{d}$, where $\sigma$ is the cross section and $d$ is the column diameter. ${ }^{31,32}$ Since the measured thermal electron temperature (Fig. 9) is fairly constant beyond $z=8 \mathrm{~cm}$ and is also constant in radius, the emission cross section will not vary substantially for $z>8 \mathrm{~cm}$. As shown in Fig. 11(b), the helicon "blue" mode emission scales with average density squared much more closely than the transition mode shown in Fig. 11(a). However, the blue mode emission does not scale as well for positions between the peak density at $z=18 \mathrm{~cm}$ and the antenna region. This implies that other processes than a Maxwellian that enhance ionization efficiency can be present in both the transition mode as well as in the blue mode between $z=18 \mathrm{~cm}$ and the antenna. Moreover, the optical modulation decreases (from $23 \%$ to $7 \%$ ) with magnetic field strength. This indicates that the electron distribution deviates less from Maxwellian as the magnetic field is increased.

The spatially constant peak emission phase observed in the higher density and magnetic field "blue" mode ${ }^{33}$ regimes has several possible explanations. Due to statistical uncertainties in the emission process and limits of the diagnostic system $(\Delta t=7.3 \mathrm{~ns} / \mathrm{bin})$, a stimulated emission pulse traveling at the speed of light would appear as a constant phase signal. Another possibility is that wave interference of multiple axial wavelength modes results in a standing wave field structure that gives rise to the constant phase signal. The wave field of Fig. 10(d) has a substantial ratio of maximum to minimum wave amplitude versus axial position, implying wave interference and a substantial standing wave component of uniform phase for the total wave field. In addition, the phase variation for the "blue" modes shown in Figs. 10 (c) and 10(d) is not linear with axial position as is the case for the transition mode in Fig. 10(b). This implies that multiple substantial $k_{z}$ component wave modes are present at the higher densities as has been indicated by our ANTENAII code runs.

\section{SUMMARY}

The wave magnetic field and non-perturbing optical emission observations for a helicon plasma as the static magnetic field is varied over a large range are presented. It is found that at low magnetic fields corresponding to the transition regime $\left(P=800 \mathrm{~W}, \quad B_{0}=200 \mathrm{G}, \quad n_{e}=2-4\right.$ $\times 10^{12} \mathrm{~cm}^{-3}$ ), the wave magnetic field, peak in the $443 \mathrm{~nm}$ optical emission modulation phase, and computed wave phase travel with a speed corresponding to an average resonant energy of $18 \mathrm{eV}$ as shown in Fig. 15. Since the substantial emission modulation $(25 \%)$ correlated with the wave phase is created by fast electrons above the thermal temperature of $4.3 \mathrm{eV}$, it indicates that a substantial fraction of the corresponding electron distribution has a wave-correlated, non-Maxwellian character, as indicated by earlier research at lower densities and magnetic fields on the WOMBAT experiment. $^{22}$

When the static magnetic field is increased from 600 to $1000 \mathrm{G}$ at $800 \mathrm{~W}$ of coupled power, the optical modulation decreases from $23 \%$ to $7 \%$ and the $443 \mathrm{~nm}$ peak emission phase appears constant with axial position. This appears as an abrupt transition from a traveling wave peak emission phase character for $B_{0}<300 \mathrm{G}$ to an axially constant peak emission phase for $B_{0}>400 \mathrm{G}$. The wave $B_{z}$ fields in this case show a stronger amplitude modulation and the phase oscillates substantially with position, indicating that the wave is due to the interference of multiple $k_{z}$ wave components. In addition, the $443 \mathrm{~nm}$ emission modulation level decreases as the static magnetic field is increased, while the 
density rises and becomes more peaked in radius. This indicates that the electron distribution has a reduced nonMaxwellian character for the high density and magnetic field cases. These results indicate that the physical character and ionization processes for highly efficient helicon plasma sources changes substantially with the experimental conditions and care must be taken to identify the mechanisms responsible for different sources and regimes of operation.

\section{ACKNOWLEDGMENTS}

We thank Ben White, Alex Degeling, and Richard Sund for useful discussions.

The research was supported by NSF and AFOSR.

${ }^{1}$ R. W. Boswell and F. F. Chen, IEEE Trans. Plasma Sci. 25, 1229 (1997).

${ }^{2}$ F. F. Chen and R. W. Boswell, IEEE Trans. Plasma Sci. 25, 1245 (1997).

${ }^{3}$ Y. Mouzouris and J. E. Scharer, IEEE Trans. Plasma Sci. 24, 152 (1996).

${ }^{4}$ Y. Mouzouris and J. E. Scharer, Phys. Plasmas 5, 4253 (1998).

${ }^{5}$ R. Kinder and M. J. Kushner, J. Vac. Sci. Technol. A 19, 76 (2001).

${ }^{6}$ G. G. Borg, J. Bright, and I. V. Kamenski, Plasma Phys. Controlled Fusion 40, 987 (1998).

${ }^{7}$ A. W. Degeling and R. W. Boswell, Phys. Plasmas 4, 2748 (1997).

${ }^{8}$ S. Cho and J. G. Kwak, Phys. Plasmas 4, 4167 (1997).

${ }^{9}$ R. T. S. Chen and N. Hershkowitz, Phys. Rev. Lett. 80, 4677 (1998).

${ }^{10}$ A. Degeling, N. Mikhelson, R. Boswell, and N. Sadeghi, Phys. Plasmas 5, 572 (1998)

${ }^{11}$ M. M. Balkey, R. F. Boivin, J. L. Kline, and E. E. Scime, Plasma Sources Sci. Technol. 10, 284 (2001).

${ }^{12}$ X. M. Guo, J. Scharer, Y. Mouzouris, and L. Louis, Phys. Plasmas 6, 3400 (1999).

${ }^{13}$ A. J. Perry, D. Vender, and R. W. Boswell, J. Vac. Sci. Technol. B 9, 310 (1991).

${ }^{14}$ G. R. Tynan, A. D. Bailey III, G. A. Campbell et al., J. Vac. Sci. Technol. A 15, 2885 (1997).
${ }^{15}$ G. Giroult-Matlakowski, C. Charles, A. Durandet et al., J. Vac. Sci. Technol. A 12, 2754 (1994).

${ }^{16}$ P. A. Keiter, E. E. Scime, and M. M. Balkey, Phys. Plasmas 4, 2741 (1997).

${ }^{17}$ M. D. Carter, F. W. Baity, Jr., G. C. Barber et al., Phys. Plasmas 9, 5097 (2002).

${ }^{18}$ J. P. Squire, F. R. Chang Diaz, T. W. Glover et al., Trans. Fusion Sci. Technol. 43, 111 (2003).

${ }^{19}$ J. P. Squire, F. R. Chang-Diaz, V. T. Jacobson et al., 15th Topical Conference on Applications of Radio frequency Power in Plasmas, 19-21 May 2003, Jackson Lake, Moran, WY [AIP Conf. Proc. 694, 423 (2003)].

${ }^{20}$ A. W. Molvik, T. D. Rognlien, J. A. Byers et al., J. Vac. Sci. Technol. A 14, 984 (1996).

${ }^{21}$ D. D. Blackwell and F. F. Chen, Plasma Sources Sci. Technol. 10, 226 (2001).

${ }^{22}$ J. Scharer, A. Degeling, G. Borg, and R. Boswell, Phys. Plasmas 9, 3734 (2002).

${ }^{23}$ A. W. Degeling, Ph.D. thesis, Australian National University, Canberra, 1999.

${ }^{24}$ O. Auciello and D. L. Flamm, Plasma Diagnostics: Discharge Parameters and Chemistry (Academic, New York, 1989).

${ }^{25}$ K. Akhtar, J. Scharer, S. Tysk, and E. Kho, Rev. Sci. Instrum. 74, 996 (2002).

${ }^{26}$ A. Ganguli, M. K. Akhtar, and R. D. Tarey, Plasma Sources Sci. Technol. 8, 519 (1999).

${ }^{27}$ A. Kimori, T. Shoji, K. Miyamoto, J. Kawai, and K. Kawai, Phys. Fluids B 3, 893 (1991).

${ }^{28}$ C. M. Franck, O. Grulke, and T. Klinger, Rev. Sci. Instrum. 73, 3768 (2002).

${ }^{29}$ V. Vujnovic and W. L. Wiese, J. Phys. Chem. Ref. Data 21, 919 (1992).

${ }^{30}$ J. E. Scharer (private communication).

${ }^{31}$ J. D. Huba, NRL Plasma Formulary (Naval Research Laboratory, Washington, DC, 2002).

${ }^{32}$ I. D. Sudit and F. F. Chen, Plasma Sources Sci. Technol. 5, 43 (1996).

${ }^{33}$ P. Zhu and R. W. Boswell, Phys. Rev. Lett. 63, 2805 (1989). 